Rev. Adm. Saúde - Vol. 18, № 71, abr. - jun. 2018

http://dx.doi.org/10.23973/ras.71.100

ARTIGO ORIGINAL

\title{
Morbimortalidade por diabetes no município de Montes Claros - MG
}

Morbimortality by diabetes in the municipality of Montes Claros - MG

\section{Tatiana Fróes Fernandes ${ }^{1}$, Maria Ivanilde Pereira ${ }^{2}$, Viviane Braga Lima Fernandes $^{3}$, Lorena Emanuelly Mendes Grilo ${ }^{4}$, Selma Ribeiro Rocha ${ }^{5}$, Antônio Gonçalves Maciel ${ }^{6}$}

1. Enfermeira, mestre em ciências da saúde. Docente do Departamento de Saúde Mental e Coletiva da Universidade Estadual de Montes Claros (Unimontes), Montes Claros, MG.

2. Graduada, doutora em ciências da saúde. Docente do Departamento de Saúde Mental e Coletiva da Unimontes, Montes Claros, MG

3. Médica, mestre em ciências da saúde. Docente do Departamento de Saúde Mental e Coletiva da Unimontes, Montes Claros, MG.

4. Enfermeira. Unimontes, Montes Claros, MG.

5. Enfermeira. Unimontes, Montes Claros, MG.

6. Sociólogo, doutor em gestão. Docente do Departamento de Saúde Mental e Coletiva da Unimontes, Montes Claros, MG.

\section{RESUMO}

Objetivo: Conhecer o perfil de morbimortalidade por diabetes no município de Montes Claros e analisar o comportamento da cobertura da Estratégia da Família (ESF) neste município no período de 2000 a 2015. Material e métodos: Trata-se de um estudo transversal, de análise longitudinal retrospectivo. A fonte de dados foi o sistema público de informações em saúde (DATASUS). Os dados sobre morbidade hospitalar foram provenientes das internações hospitalares remuneradas pelo SUS, entre 2000 e 2015, através da Autorização de Internação Hospitalar - AlH. A fonte das informações foi o 
Sistema de Informações Hospitalares do Sistema Único de Saúde (SIH-SUS). Os dados de mortalidade foram aqueles provenientes do Sistema de Informações sobre Mortalidade (SIM), de 2000 a 2014. As informações referentes à cobertura da ESF em Montes Claros foram coletadas no Departamento de Atenção Básica - DAB-MS. Resultados: Os resultados permitiram conhecer o cenário existente em Montes Claros em relação às ICSAP no grupo de diabetes mellitus. Notou-se diminuição do número de internações por essa patologia no período, ao mesmo tempo onde houve aumento da cobertura da ESF no município. Houve predomínio do sexo feminino e da faixa etária acima de 60 anos nas internações. As taxas de mortalidade por Diabetes se mostraram estáveis em Montes Claros, porém com aumento considerável em Minas Gerais. Conclusão: As análises da morbimortalidade pelas Condições Sensíveis à Atenção Primária podem contribuir para a reflexão tanto dos profissionais envolvidos em sua execução, quanto dos gestores do SUS, na busca de maior resolutividade e qualidade na assistência prestado por este nível de atenção.

Palavras-chave: atenção primária à saúde, estratégia saúde da família, indicadores de morbimortalidade, avaliação em saúde, gestão em saúde

\section{ABSTRACT}

Objective: To know the profile of diabetes morbidity and mortality in the municipality of Montes Claros and to analyze the behavior of the Family Strategy (FHS) coverage in this municipality from 2000 to 2015. Material and methods: This is a cross-sectional study longitudinal retrospective study. The data source was the public health information system (DATASUS). The data on hospital morbidity came from hospital admissions paid by SUS, between 2000 and 2015, through the Authorization of Hospital Hospitalization - AlH. The source of the information was the Hospital Information System of the Unified Health System (SIH-SUS). Mortality data were those obtained from the Mortality Information System (SIM), from 2000 to 2014. Information regarding the coverage of the FHS in Montes Claros was collected at the Department of Primary Care - DAB-MS. Results: The results allowed to know the existing scenario in Montes Claros in relation to ICSAP in the Diabetes mellitus group. There was a decrease in the number of hospitalizations due to this pathology in the period, at the same time as there was an increase in the FHS coverage in the municipality. There was a predominance of females and the age group above 60 years in the hospitalizations. The mortality rates for Diabetes were stable in Montes Claros, but with a considerable increase in Minas Gerais.

Conclusion: Morbidity and mortality analyzes by the Primary Care Sensitive Conditions can contribute to the reflection of both the professionals involved in their execution and the managers of SUS, in the search for greater resolution and quality of care provided by this level of care.

Keywords: primary health care, family health strategy, indicators of morbidity and mortality, health evaluation, health management 


\section{INTRODUÇÃO}

As doenças crônicas não transmissíveis (DCNT) representam uma ameaça para a saúde e desenvolvimento de todas as nações. A Organização Mundial da Saúde (OMS) estima que ocorra cerca de 36 milhões de mortes anuais por esse grupo de doenças, cujas taxas de mortalidade já são muito mais elevadas nos países de baixa e média renda ${ }^{1}$.

Dentre as DCNT destaca-se o diabetes mellitus (DM), que se refere a um transtorno metabólico de etiologias heterogêneas, caracterizado por hiperglicemia e distúrbios no metabolismo de carboidratos, proteínas e gorduras, resultantes de defeitos da secreção e/ou da ação da insulina. O DM vem aumentando sua importância pela sua crescente prevalência e habitualmente está associado à dislipidemia, à hipertensão arterial e à disfunção endotelial².

O DM é uma das doenças crônicas não-transmissíveis mais prevalentes no mundo. O número de diabéticos está aumentando em virtude do crescimento e do envelhecimento populacional, da maior urbanização, da progressiva prevalência de obesidade e sedentarismo, bem como da maior sobrevida de pacientes com $\mathrm{DM}^{2}$.

Prevê-se que uma epidemia de DM está em curso. Atualmente, estima-se que a população mundial com diabetes seja da ordem de 387 milhões e que alcance 471 milhões em 2035. Cerca de 80\% desses indivíduos vivem em países em desenvolvimento, onde a epidemia tem maior intensidade ${ }^{3}$.

Estima- se que no Brasil entre 2000 e 2030 o número de indivíduos com diabetes passará de 4,5 milhões para 11 milhões, sendo que a metade desta população desconhecerá o diagnóstico ${ }^{4}$.

DM é uma das principais causas de morbimortalidade em sociedades ocidentais e, apesar de políticas mundiais e reformas setoriais dos sistemas de saúde, o diabetes continua a representar um desafio para governos e sociedades, em razão da carga de sofrimento, incapacidade, perda de produtividade e morte prematura que provoca ${ }^{2}$.

Sua natureza crônica, a gravidade das complicações, e os meios necessários para controlá-las, tornam o DM uma doença muito onerosa não apenas para os indivíduos afetados e suas famílias, mas também para o sistema de saúde².

Os custos do DM afetam o indivíduo, a família e a sociedade, porém não são apenas econômicos. Os custos intangíveis (p. ex., dor, ansiedade, inconveniência e perda de qualidade de vida) também apresentam grande impacto na vida das pessoas com diabetes e seus familiares, o que é difícil de quantificar ${ }^{5}$. 
A análise epidemiológica, econômica e social do número crescente de pessoas que vivem com DM mostra a necessidade da implantação de políticas públicas de saúde que minimizem as dificuldades dessas pessoas e de suas famílias, e propiciem a manutenção da sua qualidade de vida ${ }^{6}$.

O DM faz parte de um grupo de diagnósticos que compõe as Condições Sensíveis à Atenção Primária (CSAP), ou seja, representam um conjunto de problemas de saúde para os quais a efetiva ação da atenção primária de qualidade diminuiria o risco de internações ${ }^{7}$.

Embora outros fatores possam interferir nos indicadores de internação hospitalar, a capacidade dos serviços de atenção primária na prevenção de hospitalizações desnecessárias tem sido tomada como indicador de qualidade da assistência à saúde ${ }^{8}$, apresentando-se na literatura como Internações por Condições Sensíveis à Atenção Primária (ICSAP) 7 .

A estruturação da Atenção Básica por meio da Estratégia de Saúde da Família trouxe consigo uma nova forma de enxergar e de trabalhar a saúde, contemplando as diretrizes do SUS, valorizando a integralidade e a longitudinalidade do cuidado prestado a população. A Atenção Primária a Saúde tem o papel de reconhecer o conjunto de necessidades em saúde e organizar as respostas de forma adequada e oportuna, por meio da avaliação da realidade local ${ }^{9}$.

Um grande desafio atual para as equipes de Atenção Primária é a Atenção em Saúde para as DCNT. Por se tratarem de condições prevalentes e multifatoriais, para a sua abordagem ser efetiva, é necessário o envolvimento das diversas categorias profissionais das equipes de saúde e do protagonismo dos indivíduos, suas famílias e comunidade ${ }^{10}$.

Diante deste cenário de aumento de prevalência de diabetes e do conhecimento da importância da Atenção Primária no manejo desses casos, faz-se necessário o presente estudo. Pretende-se, dessa forma, conhecer o perfil de morbimortalidade por diabetes no município de Montes Claros e analisar o comportamento da cobertura da Estratégia da Família (ESF) neste município no período de 2000 a 2015, justificando-se pelo fato de que o conhecimento do padrão de morbidade hospitalar dos serviços do Sistema Único de Saúde e da mortalidade por DM podem funcionar como subsídio para tomada de decisões de ações de promoção, prevenção e controle dos agravos crônicos desta patologia. Aliado a isso, a análise da cobertura da ESF e sua repercussão sobre a morbidade por DM na população é uma ferramenta imprescindível para dar suporte aos processos decisórios e para mensurar o impacto das ações implementadas por este serviço sobre o estado de saúde da população.

\section{MATERIAL E MÉTODOS}

Trata-se de um estudo transversal, de análise longitudinal retrospectivo. $\mathrm{O}$ recorte temporal estudado foi 2000-2015 e a unidade de análise foi o município 
de Montes Claros - MG. A fonte de dados foi o sistema público de informações em saúde (DATASUS). Os dados sobre morbidade hospitalar foram provenientes das internações hospitalares remuneradas pelo SUS, entre 2000 e 2015, através da Autorização de Internação Hospitalar - AlH. A fonte das informações foi o Sistema de Informações Hospitalares do Sistema Único de Saúde (SIH-SUS). O documento básico que alimenta esse sistema é $\mathrm{AlH}$, que habilita a internação de cada paciente e gera os valores correspondentes para pagamento do prestador. A AlH é preenchida pelo estabelecimento hospitalar e enviada mensalmente para o gestor municipal ou estadual do SUS. A consolidação dos dados no âmbito nacional dá-se no Departamento de Informática do SUS (DATASUS). Esse setor disponibiliza dados individualizados, mas não identificados, sobre o paciente e a internação, tais como gênero, idade, diagnóstico da internação, os procedimentos realizados e valores pagos.

Os dados de mortalidade utilizados neste trabalho foram aqueles provenientes do Sistema de Informações sobre Mortalidade (SIM), da Secretaria de Vigilância em Saúde do Ministério da Saúde, de 2000 a 2014. Foram analisados os óbitos por diabetes vinculadas a lista brasileira de condições sensíveis ao cuidado primário com base nos grupos de causas da classificação Internacional de Doenças CID-10.

As informações referentes à cobertura da ESF em Montes Claros foram coletadas no Departamento de Atenção Básica - DAB-MS.

Para tratamento dos dados foi utilizado o programa Tabwin 3.5 e Microsoft Excel 2003.

\section{RESULTADOS}

As taxas de internação por diabetes em Montes Claros e Minas Gerais (MG) no período de 2000 a 2015 estão apresentados na Tabela 1, onde se observa que as oscilações ao longo dos anos são mais intensas em Montes Claros, porém quando considerados o primeiro e o último ano de estudo houve uma pequena diminuição nas taxas de internação, tendo em 2000 uma taxa de 8,73 em MG e 7,16 em Montes Claros, e em 2015, uma taxa de 7,09 em MG e 5,60 em Montes Claros.

Tabela 1. Taxa de internação por diabetes mellitus por 10.000 habitantes em Minas Gerais e Montes Claros, 2000 a 2015.

\begin{tabular}{lrr} 
Ano de saída & $\begin{array}{l}\text { Taxa de internação } \\
\text { por diabetes - MG }\end{array}$ & $\begin{array}{l}\text { Taxa de internação por } \\
\text { diabetes - Montes } \\
\text { Claros }\end{array}$ \\
\hline 2000 & 8,73 & 7,16 \\
2001 & 8,08 & 5,71
\end{tabular}




\begin{tabular}{lll}
2002 & 8,21 & 5,14 \\
2003 & 8,16 & 3,76 \\
2004 & 7,44 & 3,06 \\
2005 & 7,62 & 4,35 \\
2006 & 7,31 & 4,61 \\
2007 & 7,08 & 4,09 \\
2008 & 7,40 & 5,02 \\
2009 & 7,46 & 3,99 \\
2010 & 8,12 & 4,42 \\
2011 & 7,97 & 5,98 \\
2012 & 7,85 & 5,86 \\
2013 & 8,03 & 6,69 \\
2014 & 7,68 & 6,10 \\
2015 & 7,09 & 5,60 \\
Variação & $-1,6$ & $-1,6$ \\
\hline Font: & 7,69 \\
\hline
\end{tabular}

Fonte: Ministério da Saúde. Informação Hospitalares do SUS (SIH/SUS), 2000-2015.

No Gráfico 1 estão apresentadas as taxas de internação por diabetes e a evolução da cobertura da estratégia Saúde da Família em Montes Claros nos anos de 2000 a 2015. A cobertura mostrou-se ascendente ao longo dos anos estudados, passando de $21,7 \%$ de cobertura em 2000 a $100 \%$ de cobertura em 2015. Para a frequência de internação por diabetes neste mesmo período, houve uma diminuição, passando de $7,16 \%$ para $5,6 \%$.

Gráfico 1. Evolução da cobertura da ESF em Montes Claros e a frequência de diabetes nos anos 2000-2015 


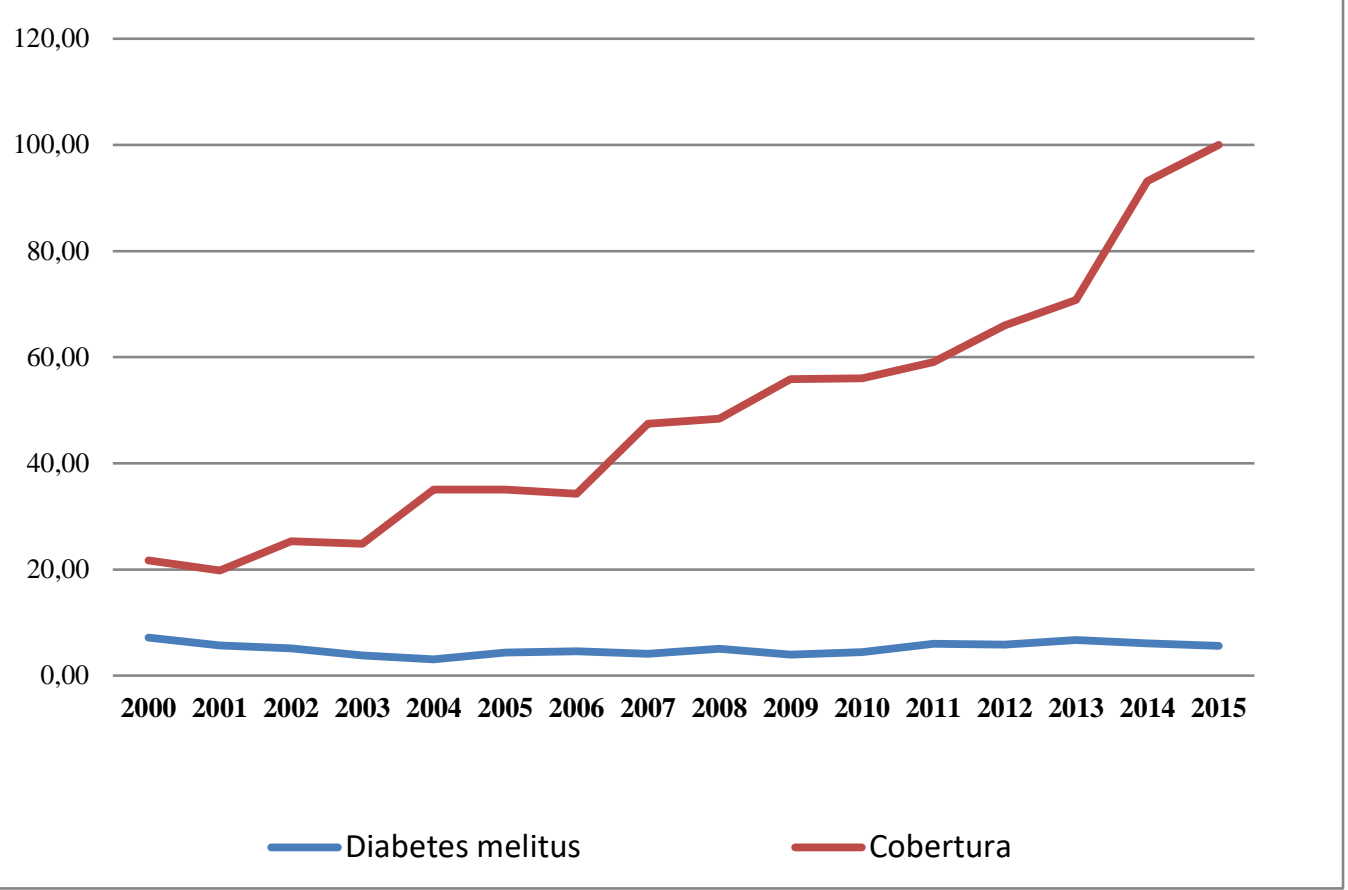

Fonte: Ministério da Saúde. Departamento de Atenção Básica/Sistema de Informação Hospitalares do SUS (SIH/SUS), 2000-2015.

Considerando a porcentagem de internações por sexo e idade, observa-se na tabela 2 que os maiores percentuais se concentraram na faixa-etária acima de 60 anos, com um aumento ao longo do tempo, passando de 32,98\% em 2000, para 39,22 em 2015. Observando a porcentagem de internações por sexo, houve predominância do sexo feminino em praticamente todos os anos desta série histórica, tendo em 2007 a maior discrepância observada no período com $32,64 \%$ das internações para o sexo masculino e $67,36 \%$ para o sexo feminino.

Tabela 2. Porcentagem de internações por diabetes mellitus, por sexo e idade, no município de Montes Claros nos anos 2000 a 2015.

\begin{tabular}{|c|c|c|c|c|c|c|c|c|c|c|}
\hline \multirow{2}{*}{$\begin{array}{l}\text { Ano de } \\
\text { saída }\end{array}$} & \multicolumn{8}{|c|}{ Idade (anos) } & \multicolumn{2}{|l|}{ Sexo } \\
\hline & $<1$ & $1-4$ & $5-9$ & $10-19$ & 20-39 & $40-49$ & 50-59 & $>60$ & $M$ & $\mathrm{~F}$ \\
\hline 2000 & 0,00 & 4,71 & 2,09 & 10,47 & 16,23 & 15,71 & 17,80 & 32,98 & 44,23 & 55,77 \\
\hline 2001 & 0,00 & 0,60 & 4,79 & 4,79 & 17,37 & 20,96 & 17,96 & 33,53 & 39,66 & 60,34 \\
\hline 2002 & 0,00 & 0,68 & 2,70 & 10,14 & 22,97 & 9,46 & 21,62 & 32,43 & 42,07 & 57,93 \\
\hline 2003 & 0,00 & 0,85 & 2,56 & 11,11 & 19,66 & 16,24 & 20,51 & 29,06 & 52,46 & 47,54 \\
\hline 2004 & 0,00 & 0,00 & 2,08 & 11,46 & 19,79 & 10,42 & 22,92 & 33,33 & 46,60 & 53,40 \\
\hline 2005 & 0,00 & 2,19 & 0,73 & 14,60 & 13,14 & 13,87 & 21,17 & 34,31 & 46,98 & 53,02 \\
\hline 2006 & 0,00 & 2,65 & 3,97 & 7,95 & 17,88 & 17,22 & 17,22 & 33,11 & 43,48 & 56,52 \\
\hline 2007 & 0,00 & 1,52 & 1,52 & 9,85 & 22,73 & 17,42 & 18,18 & 28,79 & 32,64 & 67,36 \\
\hline
\end{tabular}




\begin{tabular}{|l|r|r|r|r|r|r|r|r|r|r|}
\hline 2008 & 0,00 & 0,60 & 0,60 & 9,52 & 14,88 & 16,67 & 20,83 & 36,90 & 45,00 & 55,00 \\
\hline 2009 & 0,00 & 4,48 & 2,24 & 10,45 & 16,42 & 11,94 & 17,91 & 36,57 & 43,45 & 56,55 \\
\hline 2010 & 0,00 & 0,00 & 2,80 & 8,39 & 21,68 & 12,59 & 18,18 & 36,36 & 41,25 & 58,75 \\
\hline 2011 & 0,00 & 2,05 & 1,03 & 8,21 & 14,87 & 17,95 & 18,97 & 36,92 & 44,75 & 55,25 \\
\hline 2012 & 0,00 & 1,02 & 0,00 & 9,64 & 10,66 & 15,23 & 18,27 & 45,18 & 42,40 & 57,60 \\
\hline 2013 & 0,42 & 0,85 & 2,54 & 5,08 & 16,95 & 11,86 & 16,95 & 45,34 & 50,61 & 54,69 \\
\hline 2014 & 0,00 & 2,25 & 0,90 & 8,56 & 15,32 & 14,86 & 20,27 & 37,84 & 44,54 & 55,46 \\
\hline 2015 & 0,00 & 1,47 & 1,47 & 7,35 & 10,29 & 16,18 & 24,02 & 39,22 & 45,70 & 54,30 \\
\hline Variação & 0,00 & $-3,24$ & $-0,62$ & $-3,12$ & $-5,94$ & 0,47 & 6,22 & 6,23 & 1,47 & $-1,47$ \\
\hline
\end{tabular}

Fonte: Ministério da Saúde. Departamento de Atenção Básica/Sistema de Informação Hospitalares do SUS (SIH/SUS), 2000-2015

Com relação à mortalidade por diabetes em Montes Claros, a Tabela 3 mostra que as oscilações foram pequenas e apresentou um padrão de estabilidade, tendo no ano de 2000 uma taxa de 13,42 e em 2014, taxa de 13,58. Já a mortalidade por diabetes no estado de Minas Gerais neste mesmo período, apresentou grandes variações e um aumento significativo, passando de 18, 27 em 2000 para 24,66 em 2014.

Tabela 3. Mortalidade por causa específica de diabetes mellitus por 100.000 habitantes em Montes Claros e Minas Gerais, 2000 a 2014.

\begin{tabular}{lrr} 
Ano do óbito & $\begin{array}{l}\text { Taxa de mortalidade } \\
\text { por diabetes em } \\
\text { Montes Claros }\end{array}$ & $\begin{array}{l}\text { Taxa de mortalidade } \\
\text { por diabetes em } \\
\text { Minas Gerais }\end{array}$ \\
\hline 2000 & 13,42 & 18,27 \\
2001 & 13,39 & 16,95 \\
2002 & 14,42 & 17,20 \\
2003 & 15,72 & 18,52 \\
2004 & 13,69 & 18,61 \\
2005 & 14,01 & 19,11 \\
2006 & 13,75 & 21,11 \\
2007 & 13,34 & 22,35 \\
2008 & 15,91 & 21,27 \\
2009 & 9,09 & 21,52 \\
2010 & 17,96 & 24,77 \\
2011 & 12,29 & 24,90 \\
2012 & 11,88 & 25,88 \\
2013 & 12,18 & 24,94
\end{tabular}


Fonte: Ministério da Saúde. Departamento de Atenção Básica/Sistema de Informação sobre mortalidade (SIM), 2000-2015

\section{DISCUSSÃO}

Essa pesquisa permitiu conhecer o cenário existente em Montes Claros em relação às ICSAP no grupo de diabetes mellitus. Notou-se diminuição do número de internações por essa patologia no período 2000 a 2015, ao mesmo tempo onde houve aumento da cobertura da ESF no município. A prevalência maior de internações foi na faixa etária maior que 60 anos e no sexo feminino. As taxas de mortalidade dessa patologia no município de Montes Claros se mostraram constantes, porém houve aumento no estado de Minas Gerais, onde está inserido.

As limitações do presente estudo relacionam-se aos dados extraídos de bancos de dados de internações e óbitos do Ministério da Saúde, que, apesar de completos, podem apresentar erros relacionados ao diagnóstico registrado na autorização de internação hospitalar $(\mathrm{AlH})$, bem como, a precisão das causas descritas nos atestados de óbito e das mortes mal definidas.

Mesmo com a limitação do uso de banco de dados secundário, os resultados da pesquisa são válidos à medida que podem indicar a importância da implementação de ações no âmbito da Atenção Primária, que visam maior resolutividade e prevenção das complicações do diabetes por parte dos profissionais de saúde, e maiores investimentos nesta direção, por parte de gestores.

O estudo apontou uma redução nas taxas de internações por diabetes tanto em Montes Claros, como em Minas Gerais. A queda nas taxas de internação por diabetes também foi observada em um estudo realizado no Estado de Minas Gerais, onde reduziu de 0,85 para 0,80 internações/1.000 habitantes, entre 2000 e $2010^{11}$.

A redução nas ICSAP segue uma tendência regional e nacional, coerente com outros trabalhos, que evidenciam redução ou estabilização das internações por esse grupo de causas, apesar do aumento constante na prevalência dessas doenças em nosso meio $7,12,13,14$.

Uma análise do perfil das ICSAP atendidas pelo SUS no Brasil entre os anos de 1999 e 2007 demonstra uma redução de 24\% das ICSAP nesse período redução 2,5 vezes maior quando comparada à redução observada para outras condições ${ }^{9}$.

Estudo realizado em Minas Gerais entre 2003 a 2012 encontrou diminuição das ICSAP e chama atenção o fato dessas internações terem diminuído (111.151) em número absoluto, enquanto as não ICSAP aumentaram (65.138) no período 
considerado. As ICSAP representavam cerca de 35,97\% das internações (sem parto) em 2003 no início da série histórica. Desde então, observa-se uma constante redução na proporção de ICSAP, atingindo o percentual mais baixo em $2012(26,2 \%)^{12}$.

Dentro das CSAP, em se tratando especificamente de diabetes mellitus, observou que esse grupo de causas isoladamente, em consonância com esta pesquisa, teve diminuição no número de internações em alguns estudos realizados no país ${ }^{15,11}$. No entanto, também existem trabalhos que divergem dos achados desta pesquisa, mostrando aumento no número de internações por diabetes ao longo dos anos 16,14,17.

Este fato merece destaque, pois a assistência prestada pela atenção primária no manejo deste agravo é fundamental, tendo em vista que o diagnóstico precoce e o tratamento ambulatorial do diabetes são efetivos para evitar hospitalização e complicações potenciais ${ }^{10}$.

As divergências da frequência de internações por diabetes em diferentes regiões do país indicam os grandes desequilíbrios regionais existentes. Importantes diferenças na cobertura, no acesso e na oferta de cuidados nas Unidades Básicas de Saúde (UBS) nos municípios, são atribuídas aos mecanismos de gestão e as desigualdades sociais do país, com repercussões importantes no acesso e uso dos serviços de saúde ${ }^{18}$.

Apesar da indução do Governo Federal para a implantação de equipes de Atenção Básica, os processos organizacionais são muito variados e isto resulta em grandes contrastes locais, que afetam as coberturas, acesso aos serviços e respostas. Estas desigualdades em parte refletem as prioridades na implantação e organização e diferentes estágios de gestão e de priorização ${ }^{19}$.

Mesmo com ritmos diferentes entre as regiões, no Brasil, a implantação da Estratégia Saúde da Família experimentou grande expansão de cobertura a partir dos anos $2000^{20}$.

Em Montes Claros observou-se um aumento expressivo da cobertura populacional da ESF ao longo dos anos estudados, passando de $21,7 \% \mathrm{em}$ 2010 para $100 \%$ de cobertura em 2015. Este incremento no número de equipes também ocorreu em todo o país. No ano de 2000, as Equipes de Saúde da Família cobriam 17,43\% da população brasileira e no ano de 2012 a cobertura aumentou para $54,84 \%$, representando uma variação de 37,41 no período ${ }^{20}$.

A ESF é considerada estratégia prioritária de estruturação da atenção primária e a principal porta de entrada do SUS. A ampliação da cobertura da ESF é importante porque além de ser o primeiro contato do usuário, ele atua na longitudinalidade, na coordenação do cuidado, e como suporte dos serviços de apoio diagnóstico, assistência especializada e hospitalar ${ }^{18}$.

O aumento de cobertura da ESF pode estar associado ao destaque que é dado no SUS à atenção primária, entendendo-a como foco prioritário da organização do cuidado em saúde. Esta é uma tendência tanto brasileira como mundial e tem como objetivo diminuir o gasto hospitalar ${ }^{9}$, pois essa expansão apresenta 
forte impacto no acesso e uso da atenção primária em saúde, com potencial de reduzir as ICSAP ${ }^{13}$.

Estudo demonstra que o investimento na APS brasileira, representada, especificamente, pela ESF, esteve associado a uma redução nos recursos públicos destinados às hospitalizações em geral e às ICSAP ${ }^{21}$.

A associação entre a expansão da ESF e a diminuição da ICSAP está muito presente na literatura atual| ${ }^{12,15}$. Estudo realizado tendo como referência o Estado de Minas Gerais aponta que o modelo de cuidado implantado neste Estado por meio da ESF é de boa resolubilidade ficando confirmada a deshospitalização por condições sensíveis ao cuidado primário com a ampliação da cobertura da ESF ${ }^{12}$.

No contexto brasileiro, estudo encontrou menores taxas de internações por CSAP em regiões com maior cobertura da Estratégia Saúde da Família (ESF) e valores mais elevados de internações em regiões com maior quantidade de leitos hospitalares privados ${ }^{22}$.

Entretanto deve-se ter cautela ao analisar o comportamento das ICSAP, pois, esse indicador pode estar relacionado a um conjunto de ações, e não somente a um fator específico. Os resultados não devem ser atribuídos apenas ao nível do cuidado em saúde, mas também relacionados à ampla gama dos determinantes sociais do processo saúde-doença ${ }^{23}$.

Portanto, considera-se necessária a análise, não apenas dos resultados da assistência, por meio das internações, por exemplo, mas também do processo de trabalho e da estrutura de que os profissionais dispõem para implementar suas ações.

Em se tratando da influência do sexo nas internações por diabetes em Montes Claros, o estudo obteve predominância do sexo feminino, sendo em média $15 \%$ superior às internações do sexo masculino ao longo de todo o período estudado.

O resultado deste estudo é semelhante a de outras pesquisas sobre esse fenômeno, que evidenciam maior número de internações por CSAP em mulheres ${ }^{24,25}$.

O fato da mulher historicamente ser mais voltada ao cuidado pessoal de sua condição de saúde, pode justificar esse fato. Ela pode estar internando mais por ser mais frequente aos serviços de saúde ${ }^{26}$.

As mulheres, além das Unidades Básicas de Saúde (UBS), buscam também os serviços hospitalares, algumas vezes devido à baixa resolutividade das UBS frente aos problemas de saúde, sendo necessário o encaminhamento para outro nível de atenção ${ }^{14}$.

Estudo de análise da evolução do perfil de utilização dos serviços pelas mulheres, realizado no Brasil nos anos de 2003 a 2008, mostrou que a procura de serviços de saúde por mulheres manteve-se maior em todo o período analisado (17\% contra 10\% dos homens), além de haver observado um 
aumento geral da procura pelos serviços de saúde em função da idade: $12 \%$ na faixa de 0 a 14 anos; $14 \%$ nos 15 aos 59 anos; e 23\% entre os acima dos 60 anos $^{27}$.

A maior procura das mulheres por serviços de saúde, também demonstrada em outros estudos, faz com que elas apresentem 2,2 vezes mais chances de serem diagnosticadas com diabetes, na comparação com os homens ${ }^{28}$.

Este fato pode estar associado à demanda pelos serviços de saúde, com ênfase para a atenção primária, composta de programas que, ao beneficiarem o cuidado à saúde da mulher nos diferentes ciclos da vida, favorecem e repercutem na maior procura e utilização desses serviços, especialmente por mulheres com mais idade ${ }^{27}$.

Considerando a idade predominante nas internações por DM, o presente estudo encontrou um predomínio da faixa etária acima de 60 anos, o que ocorre com a grande maioria das doenças crônicas ${ }^{29}$. Especificamente com relação ao diabetes, estudo realizado em 2015 em São José do Rio Preto, SP, encontrou uma média de idade de 61 anos para as internações ${ }^{26}$.

Ao refletir sobre o envelhecimento populacional ora em curso, é provável que nos próximos anos ocorram cada vez mais hospitalizações por causas sensíveis à APS caso não sejam efetivadas intervenções em nível de prevenção primária e secundária. Alguns autores têm se aprofundado no estudo da morbidade existente entre idosos, na busca por medidas mais eficazes para reduzir o acometimento desse grupo ${ }^{30,31}$.

O fato dos maiores índices de internação concentrarem-se na população idosa está em concordância com a literatura, já que a forma predominante da doença, o DM tipo 2, também é predominante nesta faixa etária, além do que a progressão lenta, insidiosa e com possíveis complicações do diabetes pode culminar em hospitalização após alguns anos do diagnóstico.

A ocorrência de ICSAP em idosos, além de outros fatores, pode estar relacionada tanto às limitações de acesso mais comuns nessa população (dificuldade de locomoção e transporte, alto grau de dependência) como ao próprio envelhecimento da população brasileira, acompanhado do desenvolvimento de doenças crônicas não transmissíveis ${ }^{25}$.

Os dados do estudo ainda são condizentes com os resultados de um estudo nacional que demonstrou que $33,5 \%$ das internações hospitalares pelo SUS são de idosos e consomem $37,7 \%$ dos recursos pagos pelo sistema público de saúde ${ }^{32}$.

Ainda que todos os grupos etários sejam atingidos pelo diabetes, são as pessoas de maior idade a exigir maiores investimentos em serviços de saúde para seu tratamento, haja vista a elevada prevalência da doença nesse grupo etário ${ }^{33}$.

No que se refere às as taxas de mortalidade por diabetes (por 1000.000 habitantes), em Montes Claros foi observado um padrão de estabilidade ao 
longo do período, passando de 13,42 em 2000 e 13,58 em 2014, já no estado de Minas Gerais, houve um aumento de 18,27 em 2000 para 24,66 em 2014.

Dados brasileiros de 2011 mostram que as taxas de mortalidade por DM (por 100 mil habitantes) são de 33,7 para a população geral, 27,2 nos homens e 32,9 nas mulheres, com acentuado aumento com o progredir da idade, que varia de 0,50 para a faixa etária de 0 a 29 anos a 223,8 para a de 60 anos ou mais, ou seja, um gradiente de 448 vezes $^{34}$.

Na maioria dos países desenvolvidos, verifica-se que o DM figura entre a quarta e a oitava posição entre as principais causas básicas de óbito. Estudos brasileiros sobre mortalidade por DM, na análise das causas múltiplas de morte, ou seja, quando se menciona DM na declaração de óbito, mostram que a taxa de mortalidade por essa enfermidade aumenta até 6,4 vezes $^{35}$.

Frequentemente, na declaração de óbito, não se menciona DM pelo fato de serem suas complicações, particularmente as cardiovasculares e cerebrovasculares, as causas da morte. No início do século 21, estimou-se que se atribuíram $5,2 \%$ de todos os óbitos no mundo ao diabetes, o que torna essa doença a quinta principal causa de morte. Parcela importante desses óbitos é prematura, ocorrendo quando ainda os indivíduos contribuem economicamente para a sociedade ${ }^{36}$.

A DM causa em torno de $5 \%$ de todas as mortes no mundo, anualmente. Estima-se que, as mortes por DM aumentarão em mais de $50 \%$ nos próximos 10 anos, caso medidas urgentes não forem iniciadas ${ }^{37}$.

As condições crônicas representam um problema de saúde pública, em virtude do impacto negativo na situação de vida das pessoas acometidas e na organização dos serviços de saúde. A DM avança silenciosamente, em muitos casos, ao longo dos anos, não sendo tratada adequadamente e detectada tardiamente, repercute em sérias consequências, aumentando as taxas de internações e mortalidade. O planejamento da assistência à saúde é fundamental, desenvolvendo atividades de promoção para a saúde, prevenção de agravos, cuidado integral e longitudinal, melhorando a qualidade de vida da população e a cobertura pelas equipes de saúde ${ }^{10}$.

Diante deste cenário, abordagens sobre o conhecimento dos padrões de morbidade e mortalidade, bem como a organização, gestão e avaliação dos serviços para o cuidado das condições crônicas devem ser adotadas, colaborando para a transformação da realidade prática local.

\section{CONCLUSÃO}

Embora futuros estudos, com desenhos mais complexos, sejam necessários para avaliar as relações de causalidade aqui levantadas, a presente pesquisa aponta para uma melhoria no quadro das internações por diabetes mellitus no Município. Os dados observados foram semelhantes a outros estudos brasileiros. 
O resultado de estabilização na frequência das ICSAP no município de Montes Claros para avaliação do desempenho desse nível de atenção deve ser visto com cuidado, posto que se trata de um indicador que apresenta algumas limitações, dentre as quais a própria confiabilidade do diagnóstico e o uso de dados secundários.

Novos estudos devem ser guiados para o conhecimento do cuidado prestado aos usuários com DM no âmbito da Atenção Primária em Montes Claros, analisando a capacidade diagnóstica, o manejo dos pacientes e a estrutura dos serviços, tudo isso considerando a história natural da evolução do DM que pode culminar com internações, principalmente em idosos, apesar dos cuidados prestados mesmo de forma oportuna.

Apesar desses aspectos, estudos de avaliação das ICSAP devem ser realizados, pois se trata de um indicador que mede indiretamente 0 funcionamento e a capacidade de resolução da APS e pode contribuir para a reflexão tanto dos profissionais envolvidos em sua execução, quanto dos gestores do SUS, na busca de maior resolutividade e qualidade na assistência.

\section{REFERÊNCIAS}

1. World Health Organization.Global status report on non communicable diseases 2010. Geneva: World Health Organization [Internet]. 2011 [Cited 2016 Oct 24 ]. Available from: http://www.who.int/nmh/publications/ncd report full en.pdf

2. World Health Organization. Global report on diabetes the cost of diabetes. Geneve: WHO [Internet]. 2016 [Cited 2016 Oct 24 ]. Available from:

http://apps.who.int/iris/bitstream/10665/204871/1/9789241565257 eng.p $\underline{\mathrm{df}}$

3. International Diabetes Federation. IDF Diabetes Atlas. 6a ed. Brussels: International Diabetes Federation [Internet]. 2014. [Cited 2016 Oct 25 ]. Available from: http://www.idf.org/diabetesatlas

4. Wild S, Roglic G, Green AA, Sicree R, King H. Global Prevalence of Diabetes Estimates for the year 2000 and projections for 2030. Diabetes Care [Internet]. 2004 [Cited 2016 Sep 29]; 27(5):1047-1053. Available from: http://care.diabetesjournals.org/content/27/5/1047.full-text.pdf

5. Diretrizes da Sociedade Brasileira de Diabetes (2015-2016) / Adolfo Milech...[et. al.]; organização José Egidio Paulo de Oliveira, Sérgio Vencio - São Paulo: A.C. Farmacêutica [Internet]. 2016. [Cited 2017 May 18]. Available from:

http://www.diabetes.org.br/profissionais/images/docs/DIRETRIZES-SBD2015-2016.pdf

6. Brasil. Ministério da Saúde. Secretaria de Atenção à Saúde.

Departamento de Atenção Básica. Estratégias para o cuidado da pessoa 
com doença crônica: diabetes mellitus / Ministério da Saúde, Secretaria de Atenção à Saúde, Departamento de Atenção Básica. - Brasília: Ministério da Saúde, 2013. 160p : il. (Cadernos de Atenção Básica, n. 36)

7. Alfradique ME, Bonolo PF, Dourado I, Lima-Costa MF, Macinko J, Mendonça CS, et al. Internações por condições sensíveis à atenção primária: a construção da lista brasileira como ferramenta para medir o desempenho do sistema de saúde (Projeto ICSAP - Brasil). Cad. Saúde Pública [Internet]. 2009 [Cited 2016 Sep 08]; 25(6):1337-49. Available from: http://www.scielo.br/pdf/csp/v25n6/16.pdf

8. Starfield, B. Atenção primária: equilíbrio entre necessidades de saúde, serviços e tecnologia. Unesco- Ministério da Saúde, Brasília, DF: 2002.

9. Cardoso CS, Pádua CM, Rodrigues-Júnior AA, Guimarães DA, Carvalho SF, Valentin RF, et al. Contribuição das internações por condições sensíveis à atenção primária no perfil das admissões pelo sistema público de saúde. Rev Panam Salud Publica [Internet]. 2013 [Cited 2016 Nov 22]; 34(4):227-34. Available from:

https://scielosp.org/pdf/rpsp/v34n4/03.pdf

10. Malta DC, Silva Jr. JB. Plano de ações estratégicas para o enfrentamento das doenças crônicas não transmissíveis no Brasil e a definição das metas globais para o enfrentamento dessas doenças até 2025: uma revisão. Epidemiol Serv Saúde [Internet]. 2013 [Cited 2016 Nov 24]; 22(1):151-64. Available from: http://scielo.iec.gov.br/pdf/ess/v22n1/v22n1a16.pdf

11. Rodrigues-Bastos RM, Campos EMS, Ribeiro LC, Bastos-Filho MG, Bustamante-Teixeira MT. Internações por condições sensíveis à atenção primária, Minas Gerais, 2000 e 2010. Rev Saúde Pública [Internet]. 2014 [Cited 2016 Dec 10]; 48(6):958-967. Available from: http://www.scielo.br/pdf/rsp/v48n6/pt 0034-8910-rsp-48-6-0958.pdf

12. Maciel, A. G.; Caldeira, A. P.; Diniz, F. J. L. S. Impacto da Estratégia Saúde da Família sobre o perfil de morbidade hospitalar em Minas Gerais. Saúde Debate [Internet]. 2014 [Cited 2016 Nov 08 ]; out; n. especial (38):319-330 Available from: http://www.scielo.br/pdf/sdeb/v38nspe/0103-1104-sdeb-38-spe-0319.pdf

13. Boing AF, Vicenzi RB, Magajewski F, Boing AC, Moretti-Pires RO, Peres KG, Lindner SR, Marco Aurélio Peres. Redução das Internações por Condições Sensíveis à Atenção Primária no Brasil entre 1998- 2009. Rev Saúde Pública [Internet]. 2012 [Cited 2016 nov 08]; 46(2):359-66 Available from: http://www.scielo.br/pdf/rsp/v46n2/3709.pdf

14. Rehem TCMSB, Oliveira MRF, Amaral TCL, Ciosak SI, Egry EY. Internações por Condições Sensíveis à Atenção Primária em uma metrópole brasileira. Rev Esc Enferm USP [Internet]. 2013 [Cited 2016 
Nov 22]; 47(4):884-90 Available from:

http://www.scielo.br/pdf/reeusp/v47n4/0080-6234-reeusp-47-4-0884.pdf

15. Arruda GO, Schmidt DB, Marcon SS. Internações por diabetes mellitus e a Estratégia Saúde da Família, Paraná, Brasil, 2000 a 2012 Ciência \& Saúde Coletiva [Internet]. 2018 [Cited 2018 feb 24]; , 23(2):543-552, 2018. Available from: http://www.scielo.br/pdf/csc/v23n2/1413-8123-csc23-02-0543.pdf

16. Luciano TV, Dias JA. Internações por condições sensíveis a atenção primária em município da região Norte do Espírito Santo. Rev. Bras. Pesq. Saúde [Internet]. 2015 [Cited 2017 Dec 15]; 17(3): 23-32, jul-set. Available from: http://periodicos.ufes.br/RBPS/article/viewFile/14133/9962

17. Pazó RG, Frauches DO, Galvêas DP, Stefenoni AV, Cavalcante ELB, Pereira-Silva $\mathrm{FH}$. Internações por condições sensíveis à atenção primária no Espírito Santo: estudo ecológico descritivo no período 20052009. Epidemiol. Serv. Saúde [Internet]. 2012 [Cited 2017 Dec 15 ]; , Brasília, 21(2):275-282, abr-jun. Available from: http://scielo.iec.gov.br/pdf/ess/v21n2/v21n2a10.pdf

18. Fausto MCR, Giovanella L, Mendonça MHM, Seidl H, Gagno J. A posição da Estratégia Saúde da Família na rede de atenção à saúde na perspectiva das equipes e usuários participantes do PMAQ-AB. Saude Debate [Internet]. 2014 [Cited 2017 Dec 08]; 38(n. esp.): 13-33. Available from: http://www.scielo.br/pdf/sdeb/v38nspe/0103-1104-sdeb-38-spe0013.pdf

19. Giovanella L, Mendonça MHM, Almeida PF, Escorel S, Senna MCM, Fausto MCR, Delgado MM, Andrade CLT, Cunha MS, Martins MIC, Teixeira CP. Saúde da Família: limites e possibilidades para uma abordagem integral de Atenção Primária à Saúde no Brasil. Cien Saude Colet [Internet]. 2009 [Cited 2017 Dec 08]; 14(3):783-794. Available from: http://www.scielo.br/pdf/csc/v14n3/14.pdf

20. Brasil. Ministério da Saúde. [Internet]. Brasília: DATASUS, [Cited 2016 Oct 18]. Available from: http://dab.saude.gov.br/portaldab/historico cobertura sf.php

21. Mafra F. O impacto da atenção básica em saúde em indicadores de internação hospitalar no Brasil [dissertação]. Brasília: Universidade de Brasília [Internet]. 2011. Available from: https://portal.tcu.gov.br/lumis/portal/file/fileDownload.jsp?fileld...

22. Macinko J, Oliveira VB, Turci MA, Guanais FC, Bonolo PF, Lima-Costa MF. The influence of primary care and hospital supply on ambulatory care-sensitive hospitalizations among adults in Brazil, 1999-2007. Am J Public Health [ Internet].2011 [Cited 2016 Nov 08];101(10):1963-70. Available from: 
https://search.proquest.com/openview/00ecd574783907de536b35d5621 e3194/1 ?pq-origsite $=$ gscholar $\&$ cbl $=41804$

23. Abrego G, Garza B, Sánchez T, Rojas J. Serie sobre hospitalizaciones evitables y fortalecimiento de la atención primaria em salud: el caso de México. Banco Interamericano de Desarrollo [Internet]. 2012 [Cited 2017 Dec 15]. Available from: http://idbdocs.iadb.org/wsdocs/getdocument.aspx?docnum=37841987

24. Rehem TCMSB, Egry EY. Internações por condições sensíveis à Atenção Primária no Estado de São Paulo. Ciênc Saúde Coletiva [Internet]. [Cited 2018 Jan 23]; 2011;16(12):4755-66. Available from: http://www.scielo.br/pdf/csc/v16n12/24.pdf

25. Ferreira JBB, Borges MJG, Santos LL, Forster.

AC. Internações por condições sensíveis à atenção primária à saúde em uma região de saúde paulista, 2008 a 2010. Epidemiol

Serv. Saúde [Internet]; 2014 [Cited 2017 Dec 15]; 23(1):45-56. Available from: http://www.scielo.br/pdf/ress/v23n1/2237-9622-ress-23-0100045.pdf

26. Reis AFN, Lima JC, Beccari LM, Ribeiro RCHM, Ribeiro DF, Cesarino CB.. Tendência da morbimortalidade associada à hipertensão e diabetes em município do interior paulista. Rev. Latino-Am. Enfermagem [Internet]. 2015 [Cited 2018 Jan 25]; 23(6):1157-64. Available from: https://www.revistas.usp.br/rlae/article/view/108031/106385

27. Silva ZP, Ribeiro MCS, Barata RB, Almeida MF. Perfil sociodemográfico e padrão de utilização dos serviços de saúde do Sistema Único de Saúde (SUS), 2003- 2008. Cien Saude Colet [Internet]. 2011 [Cited 2018 Jan 25]; 16(9):3807-16. Available from:

http://www.scielo.br/pdf/csc/v16n9/a16v16n9.pdf

28. Silva DS, Laterza MC, Moreira OC, Carneiro MA, Amorim PRS.

Prevalência de diabetes mellitus em indivíduos atendidos pela estratégia saúde da família no município de Ubá-MG. Rev Bras Ativ Fis Saude [Internet]. 2012 [Cited 2017 Nov 12]; 17(3):234-42. Available from: http://rbafs.emnuvens.com.br/RBAFS/article/view/1858/1698

29. Loyola Filho Al, Matos DL, Giatti L, Afradique ME, Peixoto SV, LimaCosta MF. Causas de internações hospitalares entre idosos brasileiros no âmbito do Sistema Único de Saúde. Epidemiol Serv Saúde [Internet]. 2004 [Cited 2017 Nov 12];13(4):229-38. Available from:

http://scielo.iec.gov.br/pdf/ess/v13n4/v13n4a05.pdf

30. Magan P, Otero A, Alberquilla A, Ribera JM. Geographic variations in avoidable hospitalizations in the elderly, in a health system with universal coverage. BMC Health Services Research [Internet]. 2008 [Cited 2017 Dec 10]. Available from: http://www.ncbi.nlm.nih.gov/pmc/articles/ PMC2265697/?tool=pubmed. 
31. Valenzuela-López MI, Gastón-Morata JL, Melguizo- Jiménez M, Valenzuela-López MM; Bueno-Cavanillas A. Intervenciones sanitarias en atención primaria que disminuyen la hospitalización por Ambulatory Care Sensitive Conditions en mayores de 65 años. Atencion Primaria [Internet]. 2007 [Cited 2018 Jan 25]; 39(10):525-532 Available from: https://www.sciencedirect.com/science/article/pii/S021265670770968X

32. Peixoto SV, Giatti L, Alfradique ME, Lima-Costa MF. Custo das internações hospitalares entre idosos brasileiros no âmbito do Sistema Único de Saúde. Epidemiol Serv Saude [Internet]. 2004 [Cited 2018 Feb 26 ]; 13(4):239-46. Available from: http://scielo.iec.gov.br/pdf/ess/v13n4/v13n4a06.pdf

33. Freitas LRS; Garcia LP. Evolução da prevalência do diabetes e deste associado à hipertensão arterial no Brasil: análise da Pesquisa Nacional por Amostra de Domicílios, 1998, 2003 e 2008. Epidemiol. Serv. Saúde [Internet]. 2012 [Cited 2017 Nov 19 ]. Available from: http://scielo.iec.gov.br/pdf/ess/v21n1/v21n1a02.pdf

34. Brasil. Ministério da Saúde. Secretaria de Vigilância em Saúde. Sistema de Informações sobre Mortalidade [Internet]. Brasília: DATASUS, [Cited 2017 Sep 13]. Available from: http://www2.datasus.gov.br/DATASUS/index.php?area=060701

35. Franco LJ. Um problema de saúde pública. Epidemiologia. In: Oliveira JEP, Milech A, eds. Diabetes mellitus: clínica, diagnóstico, tratamento multidisciplinar. São Paulo: Atheneu, 2004. pp. 19-32.

36. Roglic G, Unwin N, Bennett PH et al. The burden of mortality attributable to diabetes: realistic estimates for the year 2000. Diabetes Care [Internet]. 2005 [Cited 2018 Feb 25]; 28(9):2130-5. Available from: https://www.ncbi.nlm.nih.gov/pubmed/16123478

37. Guidoni CM, Oliveira CMX, Freitas O, Pereira LRL. Assistência ao diabetes no Sistema Único de Saúde: análise do modelo atual. Bras J Pharmac Sci [Internet]. 2009 [Cited 2018 Feb 25]; 45(1):37-48. Available from: http://www.scielo.br/pdf/bjps/v45n1/05.pdf

Recebido: 27 de março de 2018. Publicado: 20 de abril de 2018

Correspondência: Tatiana Fróes Fernandes. E-mail: tatifroesn@hotmail.com

Conflito de interesses: os autores declararam não haver conflito de interesses. 
(C) This is an Open Access article distributed under the terms of the Creative Commons Attribution License, which permits unrestricted use, distribution, and reproduction in any medium, provided the original work is properly cited 